\title{
Synthesis, Characterization and Quantum Chemical Studies on a Novel Cobalt(II) 1,2,3,4-Tetra-(4-pyridyl)-thiophene Coordination Polymer
}

\author{
Jian-Guo Lin, ${ }^{\dagger \dagger}$ Ling Qiu, ${ }^{\dagger, *}$ and Qing-Jin Meng ${ }^{\ddagger}$ \\ ${ }^{\dagger}$ Key Laboratory of Nuclear Medicine, Ministry of Health \& Jiangsu Key Laboratory of Molecular Nuclear Medicine, \\ Jiangsu Institute of Nuclear Medicine, Wuxi 214063, P. R. China. "E-mail: qiulingwx@gmail.com \\ ${ }^{*}$ Coordination Chemistry Institute, State Key Laboratory of Coordination Chemistry, \\ School of Chemistry and Chemical Engineering, Nanjing University, Nanjing 210093, P. R. China \\ Received November 27, 2009, Accepted November 27, 2009
}

Key Words: Co(II) complex, Coordination polymer, Tetradentate ligand, Quantum chemical calculation, NLO property

The rational design and assembly of coordination polymers have received increasing attention and been developing rapidly in recent years. ${ }^{1}$ Being easily and efficiently constructed from relatively simple subunits, these complexes exhibit fine-tuning structural diversity and potential applications in the development of optical, electronic, and magnetic devices. ${ }^{2-4}$

It is well known that some weak interactions usually affect the structures of coordination polymers, such as hydrogen bonding and $\pi-\pi$ stacking interactions. ${ }^{5,6}$ Moreover, they can further link low-dimensional entities into high-dimensional supramolecular networks. ${ }^{7,8}$ So, the most feasible strategy used to design and construct coordination polymers is to select suitable polyfunctional ligands that enable the control on the structural motifs. ${ }^{9}$ Some tetratopic pyridyl-bridging ligands are of the recent surge of research interest, for example, 1,4-dihydroxy1,2,4,5-tetra-(4-pyridyl)-cyclohexane, 1,2,4,5-tetra-(4-pyridyl)benzene and 1,2,3,4-tetra-(4-pyridyl)-cyclobutane. ${ }^{10-14} \mathrm{Com}$ pared with the linear 4,4'-bipyridine and 4,4'-bipyridine-like $\mathrm{N}, \mathrm{N}^{\prime}$-donor ligands, ${ }^{15}$ these tetradentate ligands induce unique structural topologies and exhibit interesting physical properties in many complexes. ${ }^{10-14}$ In this field, however, the rigid symmetric ligand 1,2,3,4-tetra-(4-pyridyl)-thiophene (TPT, Scheme 1) is less explored in the self-assembly of coordination polymers. ${ }^{16}$ This ligand also possesses four potential coordinating N-donor atoms. Therefore, it can coordinate to metal centers with diverse modes.

For investigating the coordination chemistry of TPT and preparing novel materials with interesting structural topologies and excellent physical properties, we have recently been engaged in the research of polymeric complexes with this tetratopic pyridyl-bridging ligand. ${ }^{17}$ The aim of this work is to carry out a systematic investigation on the structural characterization of the $\left[\mathrm{Co}(\mathrm{TPT})_{2}(\mathrm{NCS})_{2}\right]_{\mathrm{n}} \cdot 2 \mathrm{nH}_{2} \mathrm{O}(\mathbf{1})$ polymeric complex by using

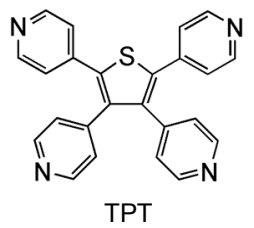

Scheme 1 experimental and theoretical methods. The IR spectrum, electronic transition property, thermal stability, and second-order nonlinear optical (NLO) property have also been studied.

The X-ray diffraction study reveals that complex 1 crystallizes in the $P 2$ space group and features a 2-D supermolecular network formed by the hydrogen bonding interactions between the water molecules and 1-D double-stranded chains. As far as we know, the coordination polymers with this chiral space group are relatively rare. ${ }^{18}$ As depicted in Figure 1, the Co1 exhibits a slightly distorted octahedral coordination sphere. The basal plane is defined by four pyridyl nitrogen atoms, while the apical positions are occupied by two nitrogen atoms from two $\mathrm{NCS}^{-}$anions. And the metal ion Co1 locates in the center of the basal plane. The distances between the metal atom and different types of nitrogen atoms in the $\mathrm{CoN}_{6}$ range from 2.078(3) to 2.2099(15) $\AA$, which are comparable to the reported values. The degrees of distortion from an ideal octahedral geometry are reflected from cisoid angles [86.74 $\left.94.61^{\circ}\right]$ and transoid angles $\left[174.4-175.7^{\circ}\right]$.

From Figure 2, one can observe that the $\mathrm{Co}(\mathrm{II})$ atoms are first connected by $\mathrm{V}$-shape bridge of the two coordinated pyridines of the TPT ligand to form 1-D ladderlike network along the $b$ axis. The nearest distance between two Co(II) centers is 9.7508 $\AA$. Besides, these 1-D chains are further connected by the strong intermolecular hydrogen bonding interaction between uncoordinationed pyridine nitrogen atom and free water molecule $(\mathrm{O} 2-\mathrm{H} 2 \mathrm{C} \ldots \mathrm{N} 42.725(2) \AA)$ to form a 2-D supramolecular net-

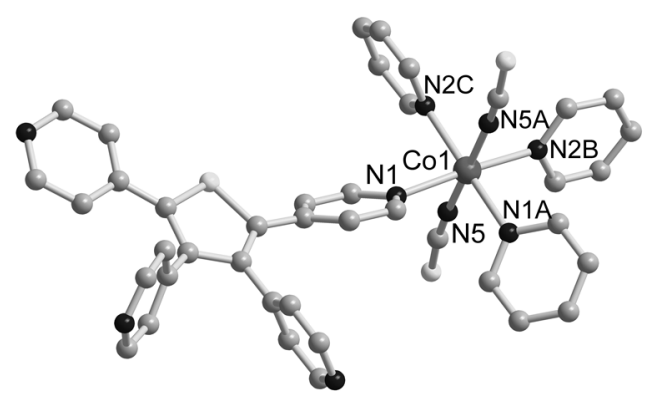

Figure 1. Molecular structure of the complex 1 (Symmetry codes are listed in Table 1). 


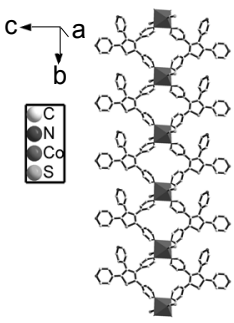

(a)

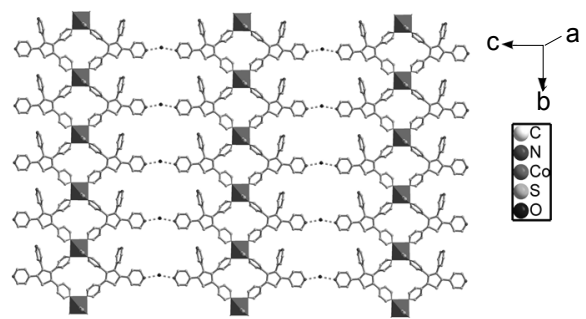

(b)
Figure 2. (a) Perspective view of $1-D$ chain of 1 connected by the TPT ligand; (b) Perspective view of 2-D supermolecular network formed by strong H-bonded interactions.

Table 1. Selected geometric parameters by X-ray single crystal diffraction and B3LYP/LANL2DZ calculation for the title complex ${ }^{a}$

\begin{tabular}{|c|c|c|c|}
\hline Parameter & Experimental & Calculated & Error $^{b}$ \\
\hline \multicolumn{4}{|c|}{ Bond lengths $(\AA)$} \\
\hline Co1-N1 & $2.2099(15)$ & 2.208 & -0.002 \\
\hline Co1-N2 & 2.194(3) & 2.222 & 0.028 \\
\hline Co1-N5 & $2.078(3)$ & 2.111 & 0.033 \\
\hline N1-C5 & 1.390 & 1.359 & -0.031 \\
\hline N5-C25 & $1.179(5)$ & 1.201 & 0.022 \\
\hline $\mathrm{C} 1-\mathrm{C} 2$ & $1.376(7)$ & 1.388 & 0.012 \\
\hline $\mathrm{C} 2-\mathrm{C} 3$ & $1.461(9)$ & 1.459 & -0.002 \\
\hline $\mathrm{C} 3-\mathrm{C} 4$ & $1.407(9)$ & 1.389 & -0.019 \\
\hline $\mathrm{C} 5-\mathrm{C} 6$ & 1.390 & 1.401 & 0.011 \\
\hline $\mathrm{C} 6-\mathrm{C} 7$ & 1.390 & 1.416 & 0.026 \\
\hline $\mathrm{C} 1-\mathrm{C} 7$ & $1.436(7)$ & 1.475 & 0.039 \\
\hline $\mathrm{C} 1-\mathrm{S} 1$ & $1.723(7)$ & 1.808 & 0.085 \\
\hline $\mathrm{C} 4-\mathrm{S} 1$ & $1.719(6)$ & 1.808 & 0.089 \\
\hline \multicolumn{4}{|c|}{ Bond angles $\left({ }^{\circ}\right)$} \\
\hline N1-Co1-N1A & $89.10(12)$ & 89.11 & 0.01 \\
\hline N1-Co1-N2B & $89.24(8)$ & 91.42 & 2.18 \\
\hline N1-Co1-N5 & $89.42(14)$ & 89.13 & -0.29 \\
\hline N1A-Co1-N2B & 175.70 & 179.44 & 3.74 \\
\hline N1A-Co1-N2C & $89.24(6)$ & 91.07 & 1.83 \\
\hline N1A-Co1-N5A & $89.42(14)$ & 89.94 & 0.52 \\
\hline N2B-Co1-N5 & $89.36(17)$ & 89.33 & -0.03 \\
\hline N2B-Co1-N5A & $86.74(15)$ & 90.26 & 3.52 \\
\hline N2C-Co1-N5A & $89.36(17)$ & 90.18 & 0.82 \\
\hline \multicolumn{4}{|c|}{ Torsion angles $\left({ }^{\circ}\right)$} \\
\hline N1-Co1-N1A-N5 & 89.35 & 89.12 & -0.23 \\
\hline N1-Co1-N1A-N5A & -94.62 & -89.65 & 4.97 \\
\hline N1-Co1-N2B-N5 & -89.43 & -89.11 & 0.32 \\
\hline N2B-Co1-N2C-N1A & 176.15 & 179.80 & 3.65 \\
\hline N2B-Co1-N2C-N5 & -89.18 & -89.30 & -0.12 \\
\hline N2B-Co1-N2C-N5A & 86.72 & 90.26 & 3.54 \\
\hline C5-N1-Co1-N2C & -74.70 & -76.31 & -1.61 \\
\hline C25-N5-Co1-N1 & -76.76 & -80.10 & -3.34 \\
\hline C25-N5-Co1-N1A & 12.28 & 9.00 & -3.28 \\
\hline C25-N5-Co1-N2C & -78.65 & -79.92 & -1.27 \\
\hline
\end{tabular}

${ }^{a}$ Symmetry codes: A: -x, y, 2-z; B: -x, 1+y, 2-z; C: x, 1+y, z. ${ }^{b}$ Error $=$ calculated value - experimental value.

work.

The optimized geometry of $\mathbf{1}$ has been obtained at B3LYP/ LANL2DZ level of theory and listed in Table 1. As seen in Table 1, most of the predicted geometric parameters are slightly larger than those experimentally determined. This is most likely due to the fact that the experimental data describe complex $\mathbf{1}$ in the solid state, whereas the calculated data correspond to the compound in the gas phase. Comparing the theoretical results with the experimental ones, it can be found that the largest difference in bond lengths occurs at the Co1-N5 bond with the discrepancy of $0.033 \AA$. Considering the bond angles, the largest deviation between the experimental and theoretical values is $3.74^{\circ}$ corresponding to the angle N1A-Co1-N2B. On the other hand, the changes of the torsion angles are larger than those of bond angles with the largest error of $4.97^{\circ}$. In general, these deviations are reasonable which occur mainly dueing to the hydrogen bonds existed in the experimental structure while the calculation only based on the related mononuclear skeleton $\left[\mathrm{Co}(\mathrm{TPT})(\text { pyridyl })_{3}(\mathrm{NCS})_{2}\right]$. Therefore, we can draw a conclusion that the optimized geometry closely resembles the crystal structure of $\mathbf{1}$ and B3LYP/LANL2DZ level can provide satisfying calculational precision for the system studied here.

The IR spectrum of the title compound shows broad peaks in high energy bands ranging from 3580 to $3200 \mathrm{~cm}^{-1}$ due to the existence of free water molecules involved in hydrogen bonds system. In the middle energy range, the typical IR absorption of $\mathrm{CN}$ is observed at $2081 \mathrm{~cm}^{-1}$ as a singlet peak. It means that the IR band of NCS groups is occurring in only one coordination metal sphere. On the low energy side of the spectrum, a series of bands are observed, $1598(\mathrm{~s}) \mathrm{v}_{\mathrm{C}=\mathrm{N}}, 1543(\mathrm{w}), 1410(\mathrm{~s})$ $v_{\text {C-s. }}$ In this case, the $\mathrm{X}$-ray diffraction analysis was allowed to assign unambiguous binding mode of the thiocyanate group.

To better understand the nature of the electronic transition property of the title complex, time-dependent density functional theory calculations and molecular orbital analyses were carried out to investigate the electron transitions in the photoluminescent process. ${ }^{19}$ According to our calculations, the lowestenergy absorption maximum for the complex 1 should be attributed to the $S_{0}-S_{4}$ transition (wavelength: $484.93 \mathrm{~nm}$; oscillator strength: 0.07 ), as the oscillator strength of the $S_{0}-S_{1}$ transition (wavelength: $545.89 \mathrm{~nm}$ ) is only 0.02 . Here, the $S_{0}-S_{1}$ transition is associated with transitions from the highest occupied molecular orbital (HOMO) to the lowest unoccupied molecular orbital (LUMO), while the dominant $S_{0}-S_{4}$ transition is associated with the transition from HOMO-1 to LUMO+1. As shown in Figure 3, the orbital HOMO-1 is thiocyanate-based and LUMO +1 is a mixture of TPT/polypyridyl $\pi^{*}$ along with metal-based orbital, so the luminescent emission of $\mathbf{1}$ is a mixed ligand-to-ligand charge transfer (LLCT) and ligand-to-metal charge transfer (LMCT) character.

Thermogravimetric analysis (TGA) for complex 1 was performed, as depicted in Figure 4. It can be noted that the first weight loss of $3.36 \%$ occurs below $60{ }^{\circ} \mathrm{C}($ calcd $3.61 \%)$, which corresponds to the loss of two free water molecules per formula. Subsequently, a plateau region is observed ranging from 60 to $337{ }^{\circ} \mathrm{C}$. Then, consecutive decomposition takes place in the temperature range of $337-462{ }^{\circ} \mathrm{C}$ attributed to the total destruction of the framework. Clearly, we can conclude that the framework of complex $\mathbf{1}$ are very stable in a higher temperature region $\left(\sim \mathrm{ca} .340^{\circ} \mathrm{C}\right)$ and worthy of further investigation as a candidate of thermally stable material. 


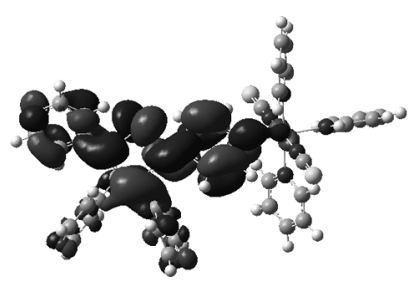

LUMO

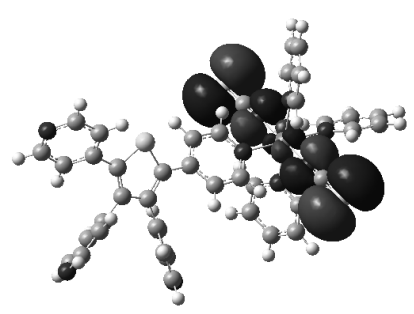

HOMO

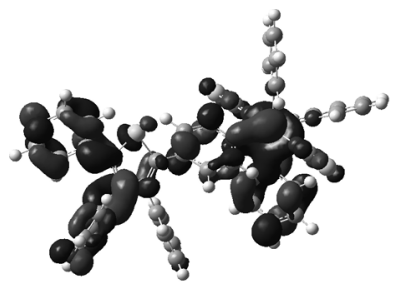

$\mathrm{LUMO}+1$

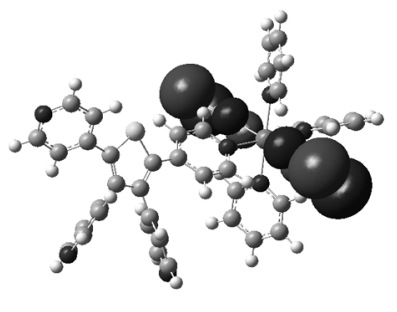

HOMO-1
Figure 3. Contour plots of the relevant molecular orbitals (from HOMO-1 to LUMO+1) in the electronic transitions of the title compound.

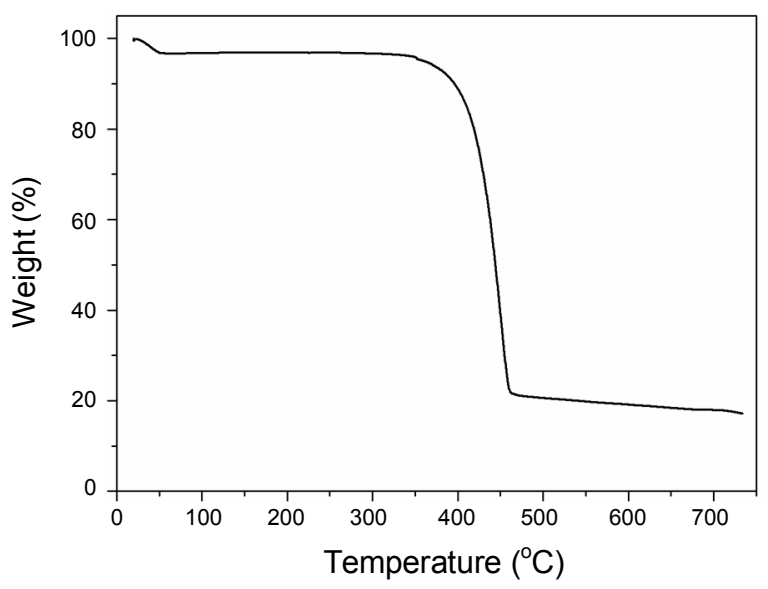

Figure 4. TGA curve of the title complex.

The chiral network is itself noncentrosymmetric, so the bulk is an acentric material that might have potential applications as NLO-active materials. ${ }^{20}$ Preliminary experimental results show that the complex 1 displays modest powder SHG efficiency, which is approximately 0.5 time that of urea. This confirms that it crystallizes in a polar space group and agrees with the structural analysis and it has potential application as the second-order NLO material.

In summary, a novel coordination polymer constructed from an interesting versatile tetradentate ligand TPT has been successfully isolated and structurally characterized. It exhibits a 1-D ladderlike network and is further connected by strong hydrogen bonding interactions to form a 2-D supramolecular network. The TPT ligand exhibits a novel coordination mode compared to the previously reported coordination polymers assembled from TPT. The skeleton of $\mathbf{1}$ is thermally stable up to a higher temperature of $337^{\circ} \mathrm{C}$ after losing the guest water molecules. In addition, complex 1 displays modest powder SHG efficiency and would be a good candidate for hybrid organic-inorganic NLO-active materials.

\section{Experimental Section}

Materials and instruments. All analytical reagents were purchased from commercial sources and used without further purification. 1,2,3,4-Tetra-(4-pyridyl)-thiophene was synthesized according to the reported work. ${ }^{17} \mathrm{C}, \mathrm{H}$, and $\mathrm{N}$ microanalyses were carried out with a Perkin-Elmer 240 elemental analyzer. The IR spectrun was recorded using $\mathrm{KBr}$ discs on a Bruker Vector 22 spectrophotometer in the $4000-400 \mathrm{~cm}^{-1}$ region. Thermogravimetric analysis was performed on a simultaneous SDT 2960 thermal analyzer under flowing $\mathrm{N}_{2}$ with a heating rate of $10{ }^{\circ} \mathrm{C} / \mathrm{min}$ between ambient temperature and $700{ }^{\circ} \mathrm{C}$. The NLO intensity was approximately estimated by measuring a powder sample of $80-150 \mu \mathrm{m}$ diameter in the form of a pellet relative to urea. A pulsed Q-switched Nd:YAG laser at a wavelength of $1064 \mathrm{~nm}$ was used to generate a secondharmonic-generation (SHG) signal from powder samples.

Calculational details. The hybrid density functional theory (DFT) B3LYP (Becke's three parameter nonlocal exchange functional along with the Lee-Yang-Parr correlation functional) ${ }^{21}$ was employed to study the complex 1 . "Double- $\xi$ " quality basis set LANL2DZ, which uses Duning D95V basis set on first row atoms and Los Alamos ECP plus DZ on Na-Bi, was employed as the basis set. The related mononuclear skeleton $\left[\mathrm{Co}(\mathrm{TPT})(\text { pyridyl })_{3}(\mathrm{NCS})_{2}\right]$ with experimental geometry was used to calculate and evaluate the structural and electronic properties of the complex 1 . All the calculations have been performed with the Gaussian 03 software package ${ }^{21}$ on a Pentium IV computer using the default convergence criteria.

Preparation of $\left[\mathrm{Co}(\mathrm{TPT})_{\mathbf{2}}(\mathrm{NCS})_{\mathbf{2}}\right]_{\mathbf{n}} \cdot \mathbf{2} \mathbf{n H}_{\mathbf{2}} \mathrm{O}(\mathbf{1})$. A solution of TPT $(0.1 \mathrm{mmol})$ in ethanol $(4 \mathrm{~mL})$ was layered over an aqueous solution of $\mathrm{Co}\left(\mathrm{NO}_{3}\right)_{2} \cdot 6 \mathrm{H}_{2} \mathrm{O}(0.1 \mathrm{mmol})$ mixed with $\mathrm{NH}_{4} \mathrm{NCS}$ $(0.2 \mathrm{mmol})$. Pink single crystals of 1 suitable for X-ray diffraction analysis were obtained by slow interlayer diffusion. The resulting crystals were filtered, and washed with water and ethanol. Yield: $37 \%$ (based on $\mathrm{Co}^{2+}$ ). Anal. Calcd for $\mathrm{C}_{50} \mathrm{H}_{36} \mathrm{Co}$ $\mathrm{N}_{10} \mathrm{O}_{2} \mathrm{~S}_{4}$ : C 60.29, H 3.64, N 14.06. Found: C 60.07, H 3.85, N $13.89 \%$.

Crystal structure analysis. $\mathrm{C}_{50} \mathrm{H}_{36} \mathrm{CoN}_{10} \mathrm{O}_{2} \mathrm{~S}_{4}, M=996.06$, Monoclinic, $P 2, a=9.2912(15), b=9.7508(16), c=12.792(2)$ $\AA, \beta=96.845(3), V=1150.7(3) \AA^{3}, Z=1, D_{\mathrm{c}}=1.437 \mathrm{~g} \mathrm{~cm}^{-3}$, $F(000)=513, R_{\text {int }}=0.0344, S=1.050, R_{1}=0.0560$, and $R_{2}=$ 0.1156 . X-ray diffraction data were collected on a Bruker SMART APEX CCD diffractometer using graphite-monochromatized Mo K $\alpha$ radiation $(\lambda=0.71073 \AA)$ using the $\omega$-scan technique. Data reductions and absorption corrections were performed with the SAINT and SADABS software packages, respectively. The Flack x parameter (absolute structure parameter) is calculated to be 0.08(3). Crystallographic data in CIF format have been deposited at the Cambridge Crystallographic Data Centre, CCDC No. 702868.

Acknowledgments. The authors are very grateful to the National Natural Science Foundation of China (20801024), Natural Science Foundation of Jiangsu Province (BK2009077), Science Foundation of Health Department of Jiangsu Province 
(H200963), China Postdoctoral Science Foundation (2008044 1026) and Jiangsu Planned Projects for Postdoctoral Research Funds (0901002B) for their financial support.

\section{References}

1. (a) Hill, R. J.; Long, D. L.; Champness, N. R.; Hubberstey, P.; Schroöder, M. Acc. Chem. Res. 2005, 38, 337. (b) Montgomery, C. P.; Murray, B. S.; New, E. J.; Pal, R.; Parker, D. Acc. Chem. Res. 2009, 42, 925.

2. Kitagawa, S.; Kitaura, R.; Noro, S. Angew. Chem. Int. Ed. 2004, 43, 2334.

3. Chung, J. W.; You, Y.; Huh, H. S.; An, B. K.; Yoon, S. J.; Kim, S. H.; Lee, S. W.; Park, S. Y. J. Am. Chem. Soc. 2009, 131, 8163.

4. An, J. S.; Geib, J.; Rosi, N. L. J. Am. Chem. Soc. 2009, 131, 8376

5. Kitagawa, S.; Vemura, K. Chem. Soc. Rev. 2005, 34, 109.

6. Lin, J. G.; Qiu, L.; Xu, Y. Y. Bull. Korean Chem. Soc. 2009, 30 , 1021.

7. Moulton, B.; Zaworotko, M. J. Chem. Rev. 2001, 101, 1629.

8. Hu, S.; He, K. H.; Zeng, M. H.; Zou, H. H.; Jiang, Y. M. Inorg. Chem. 2008, 47, 5218.

9. Cariati, E.; Macchi, R.; Roberto, D.; Ugo, R.; Galli, S.; Casati, N.; Macchi, P.; Sironi, A.; Bogani, L.; Caneschi, A.; Gatteschi, D. J. Am. Chem. Soc. 2007, 129, 9410.

10. Hu, S.; Chen, J. C.; Tong, M. L.; Wang, B.; Yan, Y. X.; Batten, S. R. Angew. Chem. Int. Ed. 2005, 44, 5471.

11. Zheng, N. F.; Bu, X. H.; Feng, P. Y. J. Am. Chem. Soc. 2002, 124 , 9688.

12. Papaefstathiou, G. S.; Milios, C.; MacGillivray, L. R. Micropor. Mesopor. Mat. 2004, 71, 11.

13. Toh, N. L.; Nagarathinam, M.; Vittal, J. J. Angew. Chem. Int. Ed. 2005, 44, 2237.
14. Papaefstathiou, G. S.; Zhong, Z.; Geng, L.; MacGillivray, L. R. J. Am. Chem. Soc. 2004, 126, 9158.

15. (a) Zaworotko, M. J. Chem. Commun. 2001, 1. (b) Biradha, K.; Fujita, M. Chem. Commun. 2001, 15.

16. Dolomanov, O. V.; Cordes, D. B.; Champness, N. R.; Blake, A. J.; Hanton, L. R. Jameson, G. B.; Schrödera, M.; Wilsona, C. Chem. Commun. 2004, 642

17. Lin, J. G.; Xu, Y. Y.; Qiu, L.; Zang, S. Q.; Lu, C. S.; Duan, C. Y.; Li, Y. Z.; Gao, S.; Meng, Q. J. Chem. Commun. 2008, 2659.

18. A search through the Cambridge Structural database (CSD version 5.30, updated to May 2009) gives the following statistical result: only 9 metal-organic coordination polymers with $P 2$ space group existed, in which no $\mathrm{Co}(\mathrm{II})$ complex has been reported.

19. Zheng, S. L.; Coppens, P. Chem. Eur. J. 2005, 11, 3583.

20. Kurtz, S. K. J. Appl. Phys. 1968, 39, 3798.

21. Frisch, M. J.; Trucks, G. W.; Schlegel, H. B.; Scuseria, G. E.; Robb, M. A.; Cheeseman, J. R.; Montgomery, J. A., Jr.; Vreven, T.; Kudin, K. N.; Burant, J. C.; Millam, J. M.; Iyengar, S. S.; Tomasi, J.; Barone, V.; Mennucci, B.; Cossi, M.; Scalmani, G.; Rega, N.; Petersson, G. A.; Nakatsuji, H.; Hada, M.; Ehara, M.; Toyota, K.; Fukuda, R.; Hasegawa, J.; Ishida, M.; Nakajima, T.; Honda, Y.; Kitao, O.; Nakai, H.; Klene, M.; Li, X.; Knox, J. E.; Hratchian, H. P.; Cross, J. B.; Adamo, C.; Jaramillo, J.; Gomperts, R.; Stratmann, R. E.; Yazyev, O.; Austin, A. J.; Cammi, R.; Pomelli, C.; Ochterski, J. W.; Ayala, P. Y.; Morokuma, K.; Voth, G. A.; Salvador, P.; Dannenberg, J. J.; Zakrzewski, V. G.; Dapprich, S.; Daniels, A. D.; Strain, M. C.; Farkas, O.; Malick, D. K.; Rabuck, A. D.; Raghavachari, K.; Foresman, J. B.; Ortiz, J. V.; Cui, Q.; Baboul, A. G.; Clifford, S.; Cioslowski, J.; Stefanov, B. B.; Liu, G.; Liashenko, A.; Piskorz, P.; Komaromi, I.; Martin, R. L.; Fox, D. J.; Keith, T.; Al-Laham, M. A.; Peng, C. Y.; Nanayakkara, A.; Challacombe, M.; Gill, P. M. W.; Johnson, B.; Chen, W.; Wong, M. W.; Gonzalez, C.; Pople, J. A. Gaussian 03, Revision C. 02. Wallingford CT: Gaussian, Inc., 2004. 